\title{
Birt-Hogg-Dubé syndrome: Report of a new mutation
}

\author{
Habib U Rehman FRCPI FRCP, FRCP(Glas) FACP
}

HU Rehman. Birt-Hogg-Dubé syndrome: Report of a new mutation. Can Respir J 2012;19(3):193-195.

Birt-Hogg-Dubé syndrome is an autosomal dominant genodermatosis caused by germline mutations in the folliculin gene and characterized by facial papules, pulmonary cysts, kidney tumours and recurrent pneumothoraces. Several distinct mutations in the folliculin gene resulting in a truncated protein have been described. The present report describes a new mutation, which has not been reported in individuals with Birt-HoggDubé syndrome but is of a type predicted to cause disease.

Key Words: Fibrofolliculoma; Folliculin; Pneumothorax; Pulmonary cysts; Renal tumour

Learning objectives

- Recognize different underlying familial causes of spontaneous pneumothorax.

- Understand the genetic basis of Birt-Hogg-Dubé syndrome.

- Comprehend the screening strategies in Birt-Hogg-Dubé syndrome.

Pre-test

- What are the clinical features of Birt-Hogg-Dubé syndrome?

- Give a differential diagnosis of familial syndromes of pneumothorax.

\section{CASE PRESENTATION}

A 47 year-old woman of French and Italian descent presented to the emergency room with increasing shortness of breath on exertion of a few days duration. She denied any chest pain, cough, fevers, orthopnea or paroxysmal nocturnal dyspnea. The patient weighed $121.5 \mathrm{~kg}$. Her height was $165 \mathrm{~cm}$ and her blood pressure was 126/88 mmHg. Multiple pale yellow-white dome-shaped nodules were seen on her face, neck and upper trunk (Figure 1). She was tachypneic. Examination of her chest revealed hyper-resonance and reduced breath sounds over the right lung. No crackles or wheezes were audible. The trachea was deviated to the left. An x-ray of the chest showed a large right pneumothorax, complete collapse of the right lung and mediastinal shift to the left. The remainder of the physical examination was unremarkable. A Heimlich valve was placed, resulting in complete expansion of her right lung.

The patient's medical history was significant for recurrent pneumothoraces. She experienced her first pneumothorax on the right side at 41 years of age, which was treated with percutaneous drainage. Six years later, she experienced two additional pneumothoraces involving the right lung, which were treated with percutaneous drainage and placement of a Heimlich valve. A previously performed computed tomography (CT) scan of the chest revealed multiple thin-walled cysts in both lungs and a cyst along the pleural surface of the right upper lobe (Figure 2). Fibrofolliculoma was diagnosed on the basis of a skin biopsy seven years previously. She was not a smoker.

\section{Le syndrome de Birt-Hogg-Dubé : rapport d'une nouvelle mutation}

Le syndrome de Birt-Hogg-Dubé est une génodermatose autosomique dominante causée par des mutations des cellules germinales du gène de folliculine et caractérisée par des papules faciales, des kystes pulmonaires, des tumeurs rénales et des pneumothorax récurrents. Plusieurs mutations distinctes du gène de folliculine responsable de la troncation d'une protéine ont été décrites. Le présent rapport décrit une nouvelle mutation, qui n’a pas été signalée chez les personnes ayant le syndrome de Birt-HoggDubé, mais qui est d'un type prédit comme responsable de la maladie.

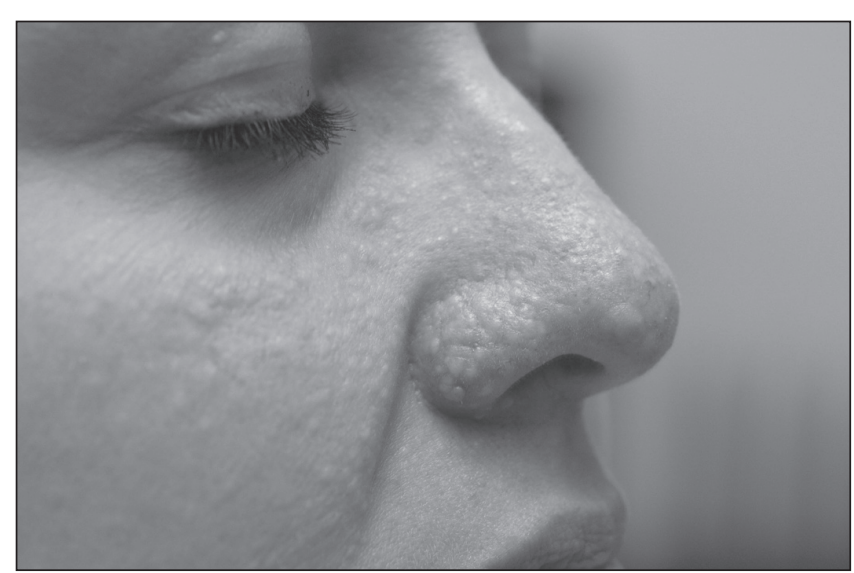

Figure 1) Facial nodules (fibrofolliculomas)

Family history was significant for facial lesions and pulmonary cysts in her father and a paternal uncle, and recurrent pneumothoraces in a paternal aunt. Her sister was diagnosed with breast cancer at 44 years of age and passed away at 60 years of age. Four maternal aunts and her maternal grandmother also had breast cancer. One paternal aunt who had a history of recurrent pneumothoraces died of cancer of the adrenal gland, and a paternal uncle died of renal carcinoma. Her paternal grandmother had died of lung cancer.

A clinical diagnosis of Birt-Hogg-Dubé syndrome (BHDS) was made. Further investigations revealed a normal CT scan of the abdomen and pelvis. Pulmonary function tests demonstrated normal spirometry, lung volumes and diffusion capacity.

A right thoracotomy was performed with wedge resection of three blebs and pleurectomy. Microscopic examination of the surgical specimen showed neutrophilic infiltration of the bleb walls. The pleural specimen also showed focal active inflammation.

Sequencing analysis of the folliculin (FLCN) gene identified one copy of a c.1117C $\rightarrow \mathrm{T}$ (p. Q373X) nonsense mutation.

\section{DISCUSSION}

BHDS is an autosomal dominant genodermatosis caused by germline mutations in the FLCN gene and characterized by facial papules, 


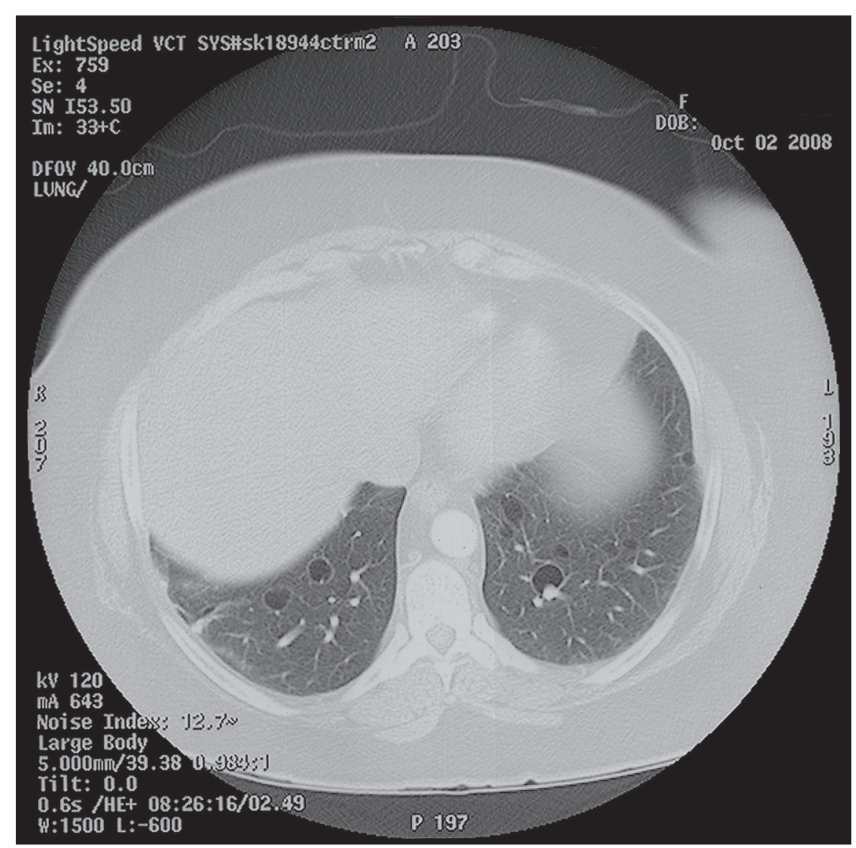

Figure 2) Computed tomography scan demonstrating multiple cysts in both lung fields

pulmonary cysts, kidney tumours and recurrent pneumothoraces. The genetic defect responsible has been mapped to a gene on chromosome 17p11.2, which encodes a tumour suppressor protein, folliculin (FLCN) (1). Folliculin is a protein expressed in a wide variety of tissues including skin, type-1 pneumocytes of the lungs and the distal nephron of the kidneys (2). Reduced expression of FLCN has been seen in renal tumours from patients with BHDS.

The skin lesions, which are fibrofolliculomas, trichodiscomas and acrochordons, typically appear as firm, yellow-white, dome-shaped papules during the third and fourth decades of life. They appear predominantly on the face, scalp, neck and upper chest.

Most patients with BHDS have radiological evidence of pulmonary cysts and/or bullae. In the largest and most comprehensive study of individuals and families with BHDS, $89 \%$ of patients had multiple pulmonary cysts (3). BHDS patients have a 32-fold greater risk of spontaneous pneumothorax than an age-matched general population. Men and women are affected equally, and smoking does not seem to be a risk factor for spontaneous pneumothorax. Spontaneous pneumothorax may be the first presentation of BHDS. It may also be the only manifestation of BHDS in some patients.

Renal tumours are found in $15 \%$ of the patients, and may be clear cell carcinoma, papillary renal cell carcinoma, oncocytomas, chromophobe renal cell carcinoma or hybrid oncocytic/chromophobe tumours (4). BHDS confers a seven-fold increased risk of developing renal neoplasia.

Colon and breast cancers might also be rare components of the spectrum of tumours associated with BHDS (5). The presence of colon cancer and colonic polyposis has been reported in several families with BHDS. However, an epidemiological study (6) showed no association between BHDS and colon cancer or polyps. More recently, colorectal polyps were identified in $50 \%$ of BHDS patients analyzed (7).

BHDS should be differentiated from other syndromes in which cystic lung disease and pneumothorax are features. These include Marfan's syndrome, homocystinuria, Ehlers-Danlos syndrome type IV, Langerhans' cell granulomatosis (LCG), tuberous sclerosis complex (TSC) and lymphangioleiomyomatosis. TSC may sometimes be difficult to distinguish from BHD. Renal angiomyolipomas, epithelial cysts, polycystic kidney disease and renal-cell carcinomas may develop in patients with TSC. Cysts in BHDS, LCG and lymphangioleiomyomatosis are all thin walled. They are found in all lung zones in lymphangioleiomyomatosis and TSC, and have a basal and subpleural prominence in BHDS, and are found in the upper and middle zones with sparing of costophrenic recesses in LCG (8).

More than 40 mutations in the FLCN gene in 200 families with BHDS have been reported worldwide (9). Eighty-four per cent of the families with BHDS have a germline FLCN mutation (10). Most of these mutations are frame-shift or nonsense mutations that lead to protein truncation (11)

Molecular analysis of the FLCN gene was performed in our patient. Sequence analysis of the entire coding region of the FLCN gene identified one copy of a c.1117C $\rightarrow \mathrm{T}(\mathrm{p} . \mathrm{Q} 373 \mathrm{X})$ nonsense mutation in exon 10 . The $c .1117 \mathrm{C} \rightarrow \mathrm{T}$ mutation is predicted to result in the replacement of the codon for the amino acid glutamine with a premature translation stop at codon 373 (p.Q373X). This mutation has not been reported in individuals with BHDS but is of a type predicted to cause disease.

The treatment of spontaneous pneumothorax in BHDS is the same as for any patient with spontaneous pneumothorax. Given the high recurrence of pneumothorax, surgical intervention with resection and pleurodesis would be an acceptable option even in patients with a first episode of pneumothorax.

Patients should be cautioned about the increased risk of pneumothorax with scuba diving and air travel due to ambient pressure effects. Screening for renal tumours and careful follow-up should be performed. An estimated $25 \%$ of FLCN mutation carriers older than 20 years of age do not manifest skin lesions, and underlying FLCN mutations have been detected in families with apparently nonsyndromic pneumothoraces. Therefore, patients with familial pneumothorax without skin lesions or other evidence of BHD should undergo CT scan of the lungs and testing for FLCN gene mutations if multiple cysts are found in the basal lung regions (11). Annual mammography for breast cancer screening is recommended. Ophthalmological and dermatological assessment and follow-up should be arranged for ocular and cutaneous melanoma screening. A susceptibility to colonic polyps and colon cancer should be taken into account in the clinical follow-up. Family members should also receive genetic counselling.

\section{Post-test}

1. BHDS is characterized by facial papules, pulmonary cysts, kidney tumours and recurrent pneumothoraces. The skin lesions, which are fibrofolliculomas, trichodiscomas and acrochordons, typically appear as firm, yellow-white domeshaped papules predominantly on the face, scalp, neck and upper chest. Most patients with BHDS have radiological evidence of pulmonary cysts and/or bullae. BHDS patients have a 32-fold greater risk of spontaneous pneumothorax than the age-matched general population. Spontaneous pneumothorax may be the first presentation of BHDS. It may also be the only manifestation of BHDS in some patients. Renal tumours are found in $15 \%$ of the patients and may be clear cell carcinoma papillary renal cell carcinoma, oncocytomas, chromophobe renal cell carcinoma and hybrid oncocytic/chromophobe tumors. BHDS confers a seven-fold increased risk of developing renal neoplasia.

2. BHDS should be differentiated from other syndromes of which cystic disease and pneumothorax are features. These include Marfan's syndrome, homocystinuria, Ehlers-Danlos syndrome type IV, LCG, TSC and lymphangioleiomyomatosis. TSC may sometimes be difficult to distinguish from BHDS. Renal angiomyolipomas, epithelial cysts, polycystic kidney disease, and renal-cell carcinomas may develop in patients with TSC. Cysts in BHD, LCG and lymphangioleiomyomatosis are all thin walled. They are found in all lung zones in lymphangioleiomyomatosis and TSC, have a basal and subpleural prominence in BHDS and are found in upper and middle zones with sparing of costophrenic recesses in LCG. 


\section{REFERENCES}

1. Schmidt LS, Warren MB, Nickerson ML, et al. Birt-Hogg-Dube syndrome, a genodermatoses associated with spontaneous pneumothorax and kidney neoplasia, maps to chromosome 17p11.2. Am J Hum Genet 2001;69:876-82.

2. Warren MB, Torres-Cabala CA, Turner ML. Expression of Birt-Hogg-Dube gene mRNA in normal and neoplastic human tissues. Mod Pathol 2004;17:998-1011.

3. Toro JR, Pautler SE, Stewart L, et al. Lung cysts, spontaneous pneumothorax, and genetic associations in 89 families with Birt-Hogg-Dubé syndrome. Am J Respir Crit Care Med 2007;175:1044-53.

4. Zbar B, Alvord WG, Glenn G, et al. Risk of renal and colonic neoplasms and spontaneous pneumothorax in the Birt-Hogg-Dube syndrome. Cancer Epidemiol Biomarkers Prev 2002;11:393-400.

5. Palmirotta R, Savonarola A, Ludovici G, et al. Association between Birt Hogg Dubé syndrome and cancer predisposition. Anticancer Res 2010;30:751-7.

6. Zbar B, Alword WG, Glenn G, et al. Risk of renal and colonic neoplasms and spontaneous pneumothorax in the Birt Hogg Dubé syndrome. Cancer Epidemiol Biomarkers 2002;11:393-400.
7. Kluger N, Giraud S, Coupier I, et al. Birt Hogg Dubé syndrome: Clinical and genetic studies of 10 French families. Br J Dermatol 2010;162:527-37.

8. Grant LA, Babar J, Griffin N. Cysts, cavities and honey combing in multisystem disorders: Differential diagnosis and findings on thin section CT. Clin Radiol 2009;64:439-48.

9. Toro JR, Wei M-H, Glenn GM, et al. BHD mutations, clinical and molecular genetic investigations of Birt-Hogg-Dubé syndrome: A new series of 50 families and a review of published reports. J Med Genet 2008;45:321-31.

10. Menko FH, van Steensel MAM, Giraud S, et al; European BHD Consortium. Birt-Hogg-Dubé syndrome: Diagnosis and management. Lancet Oncol 2009;10:1199-206.

11. Schmidt LS, Nickerson ML, Warren MB, et al. Germline BHDmutation spectrum and phenotype analysis of a large cohort of families with Birt-Hogg-Dubé syndrome. Am J Hum Genet 2005;76:1023-33.

The Canadian Respiratory Journal is now accepting submissions for a new Clinico-Pathologic Conferences series. These will be based on case presentations that illustrate important learning issues involving diagnosis and/or management decisions, and should be supported by images from appropriately applied diagnostic and/or prognostic testing which could include: 1) Lung function tests; 2) Exercise testing; 3) X-rays or computed tomography scans; 4) Ultrasound (including endobronchial ultrasound); 5) Positron emission tomography scans; or 6) Bronchoscopy/thoracoscopy.

All case reports appearing in the Canadian Respiratory Journal will conform to this format and manuscripts should be structured as described in the Instructions to Authors. A maximum of four images can be submitted and the number of references should not usually exceed 10 . The submission will be peer reviewed and may be edited by our editorial team. 


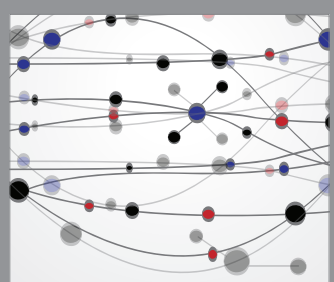

The Scientific World Journal
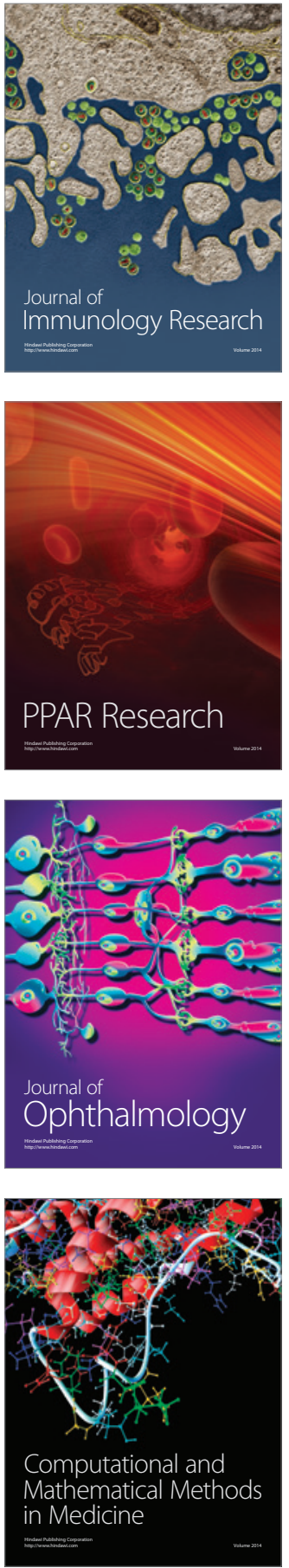

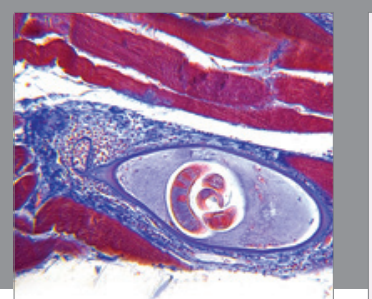

Gastroenterology Research and Practice

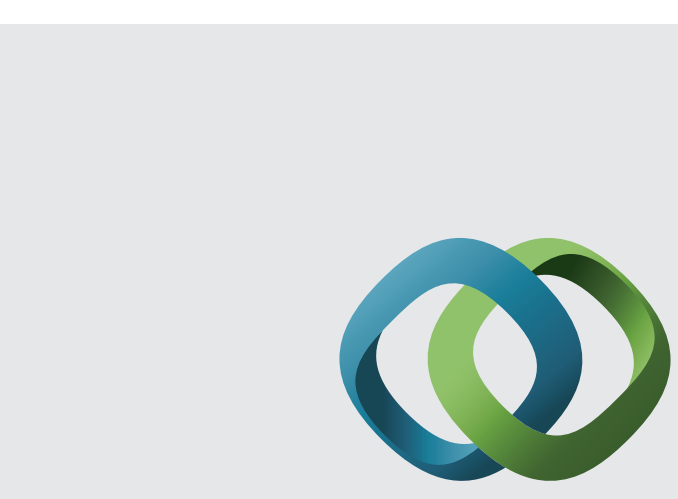

\section{Hindawi}

Submit your manuscripts at

http://www.hindawi.com
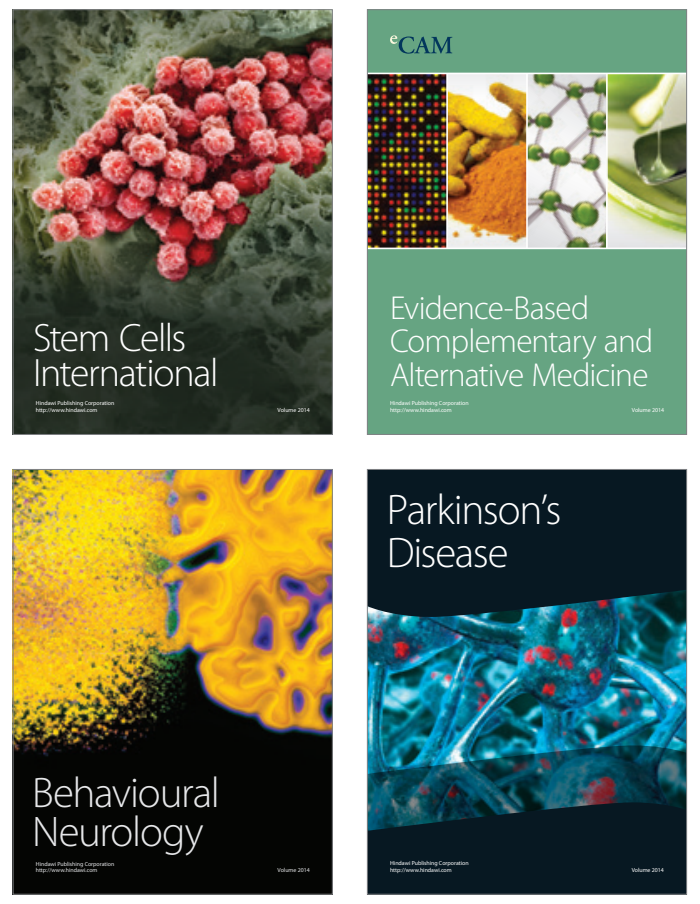
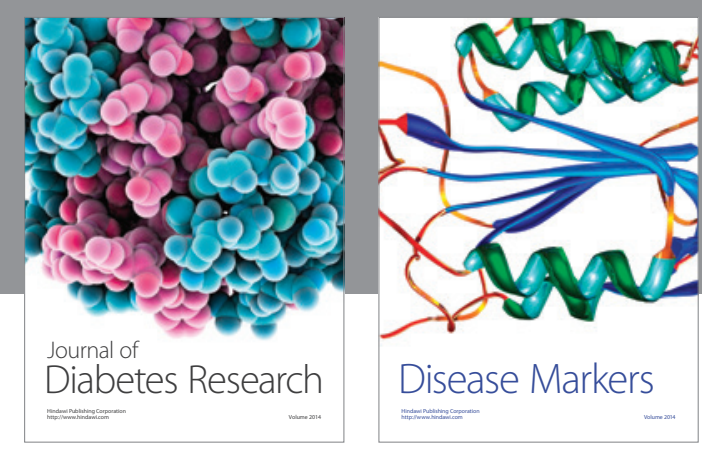

Disease Markers
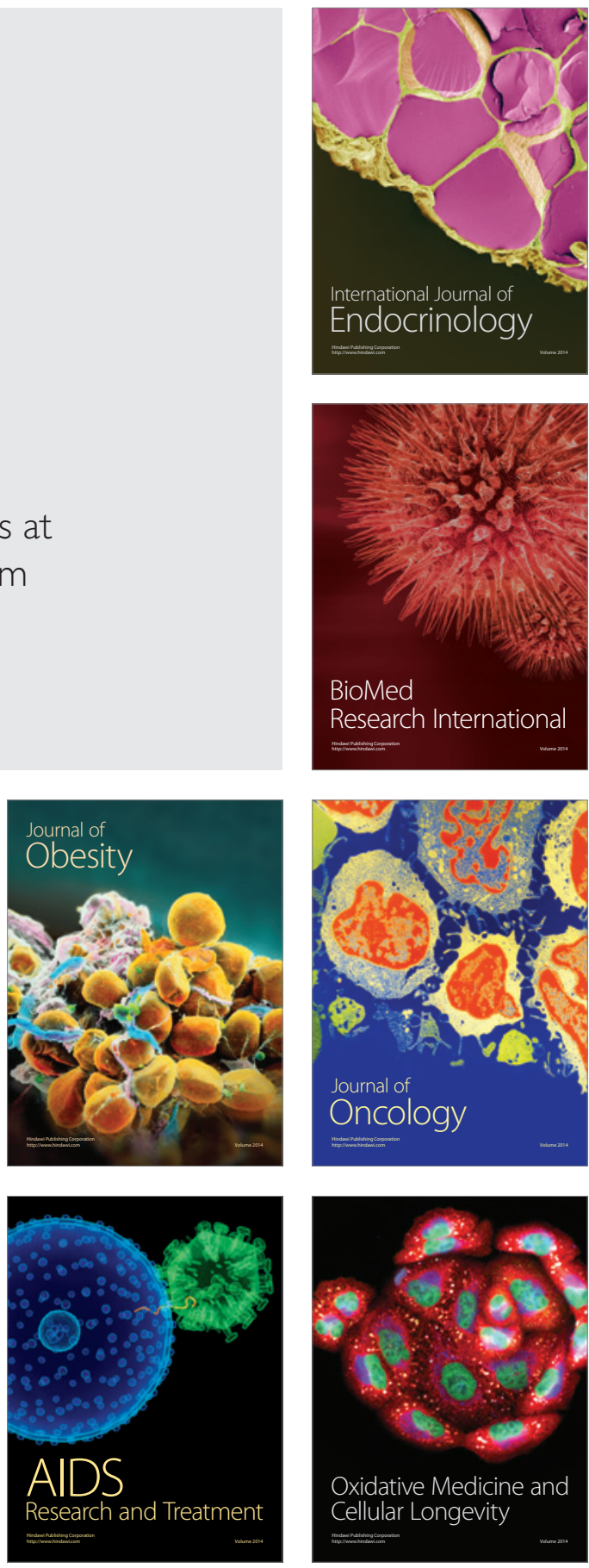